\title{
Relação do Processamento Óptico, Neurovisual e Cognitivo nas Dificuldades de Leitura
}

\author{
Dra. Márcia Reis Guimarães ${ }^{1}$, MSc Douglas de Araújoº \\ Dr. Ricardo Queiroz Guimarães ${ }^{3}$ \\ Laboratório de Pesquisa Aplicada à Neurovisão - LAPAN \\ Universidade Federal de Minas Gerais / Hospital de Olhos de Minas Gerais
}

\begin{abstract}
Resumo - Revisão integrativa dos processos ópticos, neurovisuais e cognitivos envolvidos na leitura de textos de línguas alfabéticas com o objetivo de auxiliar o diagnóstico e tratamento de pacientes com dificuldade de leitura. Para uma leitura mais eficiente e confortável, recomenda-se para alguns leitores treinamento de movimentos oculares sacádicos, adequação da iluminação do ambiente, uso de lâminas ou de filtros espectrais, uso da fonte Courier New com tamanho 14, e o consumo de ômega-3 e ômega-6 na alimentação diária. Quanto ao processo cognitivo, um bom leitor deve possuir adequada rota fonológica para eficiente conversão grafema-fonema, que por sua vez fortalece e consolida a arquitetura léxico-semântica. Intervenções para melhorar a performance da rota fonológica envolvem o ensino explicito do princípio alfabético, o conhecimento dos sons das letras e jogos de consciência fonológica. Traçou-se um paralelo entre a Síndrome de Irlen e a Dislexia com o intuito de auxiliar no diagnóstico diferencial e no prognóstico.
\end{abstract}

Palavras-chave: distúrbios da visão; leitura; dislexia; aptidão para a leitura; percepção visual; dificuldade na leitura.

\section{Relation between Optical, Neurovisual and Cognitive Processing in Reading Difficulties}

Abstract - Integrative review of optical, neurovisual and cognitive processes involved in reading of alphabetic languages texts, aiming to help the appropriate diagnostic and treatment of patients with reading difficulties. For a more efficient and comfortable reading it is recommended, for some readers, a saccadic eye movements training, adjustment of ambient light, use of spectral overlays or ophthalmic filters, use Courier New font with size 14, and intake of omega-3 and omega-6 in the daily diet. As for the cognitive process, a good reader must have a robust phonological route to efficiently make grapheme-phoneme conversion, which in turn strengthens and consolidates the lexical-semantic architecture. Interventions to improve the performance of phonological route involves the explicit teaching of the alphabetic principle, knowledge of the sounds of letters and phonological awareness games. It was drawn a parallel between the Irlen Syndrome and Dyslexia to help the differential diagnosis and prognosis.

Keywords: vision disorders; reading; dyslexia; reading readiness; visual perception; difficulties in reading.

\footnotetext{
Graduada em Medicina pela Universidade Federal de Minas Gerais (UFMG), Especialista em Oftalmologia pela Faculdade de Ciências Médicas, Mestre em Biologia Molecular pela Universidade de Paris-V, Doutora em Oftalmologia pela UFMG. Fellowship pela Moorfields Eye Hospital e pela Armed Forces Institute of Pathology.

${ }^{2}$ Mestre em Desenvolvimento Humano (Cognição e Linguagem) pela UFMG. Graduado em Psicologia pela UFMG com parte realizada na University of Leeds. douglasvilhena@gmail.com

${ }^{3}$ Graduado em Medicina, Especialização, Residência Médica e Doutorado em Oftalmologia pela UFMG. Fellowship pela Hospices Civils de Strasbourg, Moorfields Eye Hospital, Hotel Dieu de Paris e Georgetown University.
} 


\section{INTRODUÇÃO}

A leitura é considerada por muitos uma habilidade simples e automática, pois geralmente a realizamos sem esforço. Entretanto, deve-se ressaltar que ler é a habilidade cerebral mais difícil e complexa que temos, necessitando de alta qualidade e sintonia visual e cognitiva. Como o primeiro sistema escrito foi criado há apenas 5.500 anos atrás, tendo iniciado a alfabetização em massa somente no século XIX, o cérebro teve de se adaptar para responder aos ajustes requeridos neste novo sistema, por não ter ocorrido estruturalmente uma evolução específica para a leitura (DEHAENE, 2012; VILHENA, 2015). Além disso, os transtornos relacionados a leitura não possuíam nenhuma significância para a seleção evolutiva, o que explica os atuais altos índices de transtornos específico de leitura, que torna desafiador e desgastante o ato de ler para crianças e adultos.

Diferente da linguagem oral, que é adquirida naturalmente desde a vida intrauterina (MCGUINNESS, 2006), a leitura deve ser aprendida de forma sistemática. Ao se ler, as informações visuais percorrem longos trajetos em nosso cérebro, sendo moduladas em diversas regiões neuronais até serem finalmente trazidas para a consciência. Embora os olhos sejam a externalização do cérebro, tendo ambos a mesma origem embriológica, pouca atenção ainda é dada a essa relação. Essa abordagem neurovisual não faz, habitualmente, parte da formação dos profissionais da saúde, como os oftalmologistas e psicólogos, sendo excluída por não a considerarem como pertencente às suas áreas de atuação. Essa limitação restringe o acesso dos pacientes às melhores opções de tratamento, recuperação, momento ideal para intervenção e progressão acadêmica. O objetivo do presente trabalho é discutir a relação dos processos ópticos e neurovisuais que culminam no processamento cognitivo da leitura, enfatizando a importância de diagnósticos diferenciais e da pluralidade do tema para profissionais de diferentes áreas de atuação e para a ciência da educação.

\section{DESENVOLVIMENTO}

\subsection{Processamento óptico da visão}

Primeiramente, a luz, que é a matéria prima do senso visual, incide sobre o texto, sendo então refletida até alcançar os olhos. Após passar pela córnea, ocorre o ajuste da quantidade de luz pela pupila, a convergência dos raios luminosos pelo cristalino e a travessia destes pelos meios intraoculares até o fundo do olho, onde ocorre a captação da luz pela retina. A retina, camada especializada na recepção e tratamento 
inicial dos raios luminosos, é composta por três camadas conectadas em sequência que contém dezenas de tipos de neurônios com atribuições altamente específicas, onde se destacam os fotorreceptores (cones e bastonetes), os neurônios bipolares e os ganglionares. A função primordial da retina é converter a energia do espectro de luz visível em potenciais de ação, que são a linguagem de trabalho e comunicação das células nervosas e a forma de transmissão dos impulsos gerados continuamente até o Sistema Nervoso Central. A condução destes sinais se faz por milhares de axônios contidos nos respectivos nervos ópticos dos olhos direito e esquerdo, tal como ocorre com uma fibra óptica em uma transmissão digital.

Assim, como pré-requisito de uma boa leitura, é importante que haja boa qualidade de iluminação ambiental e, caso necessário, o acréscimo de um foco de luz direcionado para o texto. A fadiga frequente e progressiva que se manifesta ao se ler pode não se relacionar a problemas visuais ou cognitivos, mas sim à uma circunstancial baixa luminância, que induz resposta hormonal pela glândula pineal via melatonina, causando sono e perda de concentração. Do lado oposto, o excesso de luz pode levar a dores nos olhos e de cabeça, que são mecanismos fisiológicos de defesa para nos forçar a descansar.

Com relação ao olho em si, distúrbios genéticos, doenças, infecções ou insultos físicos podem comprometer qualquer um de seus componentes anatômicos, desde a musculatura extrínseca à córnea, cristalino, vítreo, retina, nervo óptico entre outros, interferindo com a captação e tratamento da informação visual inicial. Os principais problemas que afetam a leitura, nesse primeiro momento de captação da informação do ambiente, são os erros refracionais, como a miopia (dificuldade em focalizar objetos afastados), a hipermetropia (dificuldade em focalizar objetos próximos), o astigmatismo (falha na focalização do objeto), a presbiopia (baixa flexibilidade da acomodação do cristalino) e a catarata (aumento da opacidade do cristalino). Quando não tratados, provocam perda da nitidez da imagem ou de foco, produzindo dor de cabeça, vista embaçada, falta de concentração e o desinteresse pela leitura. Felizmente, esses problemas são detectados nos exames oftalmológicos tradicionais e solucionados com a adoção do uso de óculos de lentes corretivas, lentes de contato oftálmicas ou cirurgias. Tanto para a presbiopia (frequente após os 40 anos de idade) quanto para a catarata (frequente em idosos) é indicado o uso da fonte Courier New com tamanho mínimo 14 para melhorar o conforto durante a leitura e 
melhor delimitar o espaçamento entre as letras (MANSFIELD et al., 1996; BORRELLI et al., 2010).

A miopia, que tem início principalmente na infância e adolescência, apresenta uma prevalência cada vez maior na população mundial. Estudo realizado nos EUA verificou que houve um grande aumento na incidência de miopia nos anos de 1971-1972 (25.0\%) em comparação com 1999-2004 (41.6\%) (VITALE et al., 2009). Esta constatação seria um efeito colateral do nível de escolaridade, exposição à luz artificial e demanda visual progressiva pelo uso de tablets, celulares e tempo gasto com a leitura, elevando o esforço visual (LIN et al., 1999; SHERWIN et al., 2012; MIRSHAHI et al., 2014).

Além dessa questão de captação e tratamento da informação visual inicial, o ato de ler exige que o sistema de movimentação ocular se comporte com sincronia e de forma rígida, pois os olhos devem se mover juntos e em um sentido horizontal da esquerda para a direita. Dentre os movimentos dos olhos, os mais importantes são os de fixação e os sacádicos. As fixações são pequenas paradas para melhor captar os estímulos visuais. Ao se fixar um determinado ponto, a imagem da palavra (ou suas letras e sílabas) é captada pela fóvea, que é a região na retina com a mais alta concentração de cones, que são neurônios sensíveis aos comprimentos eletromagnéticos do espectro da luz visível. Estes estímulos eletromagnéticos são convertidos em impulsos geradores de informações com alto grau de detalhamento relacionadas à acuidade visual, garantindo desta maneira as condições ideais para início do processo de leitura, que é a decodificação da informação visual (ou dos símbolos gráficos).

Já os movimentos sacádicos são movimentos em salto feitos pelos olhos, importantes para acompanhar objetos que se movem rapidamente e também para cobrir rapidamente todo o campo visual. Para isto, os olhos se fixam (voluntária ou involuntariamente) em um ponto e rapidamente mudam para outro ponto de fixação. Esse processo ocorre durante toda a leitura sem que seja necessário movimentar a cabeça. Graças aos movimentos oculares constantes e fixações de milissegundos, todas as palavras e frases do texto são captadas.

É esperado que um leitor fluente tenha um reduzindo número de fixações, consequentemente menos movimentos sacádicos, pois emprega menos tempo na leitura e mais energia cognitiva para compreender o texto. Por exemplo, um adulto precisa de 80 a 100 fixações para ler 100 palavras (TAYLOR ASSOCIATES, 2006). Já uma criança no início da alfabetização precisa de duas fixações ou mais para ler apenas uma 
palavra, pois ainda precisa dividi-la em partes, logo, não conseguindo realizar o reconhecimento global da palavra. Leitores com movimentos sacádicos disfuncionais e excesso de fixações podem melhorar o seu desempenho com treinamentos sacádicos (CIUFFREDA et al., 1983; LEONG et al., 2014) e pelo uso de lâminas ou de filtros espectrais (SOLAN et al., 1998; LIGHTSTONE et al., 1999; ALLEN et al., 2012). 


\subsection{Processamento visual}

A visão vai muito além do globo ocular, de suas patologias e de seus erros refracionais. Após a luz ser convertida em impulsos nervosos, a informação será modulada em diferentes regiões cerebrais, precedendo a construção da imagem sensorial final, que é a informação conscientemente apreciada como visão. É importante destacar que a visão é uma habilidade cerebral abrangente, pois provê aproximadamente $70 \%$ de todas as fibras de input sensoriais para o cérebro, onde 32 áreas corticais relacionadas a visão processam informações relacionadas ao contraste, cor, movimento, direcionamento, texturas, tridimensionalidade, contextualização, memorização, entre outros (FELLEMAN; VAN ESSEN, 1991).

De forma sucinta, os impulsos nervosos que saem da retina são transmitidos pelos nervos ópticos até o quiasma óptico, onde são redistribuídos por meio dos tratos ópticos, atingindo o corpo geniculado lateral (localizado no tálamo). Esta região subcortical emite axônios que atingem o córtex visual primário no lobo occipital, via trato genículo-calcarino, localizado nas bordas do sulco calcarino. Importante destacar que a informação visual não é utilizada somente para a visão, pois aproximadamente 20\% das fibras que saem do quiasma óptico penetram no núcleo pré-tectal (nocicepção e movimentos oculares reflexivos) ou no hipotálamo supraquiasmático (regulação dos ritmos circadianos) (COSENZA, 2005).

Ao cruzar o quiasma óptico, metade das fibras de cada um dos nervos ópticos se cruza em direção ao lado oposto do cérebro. Assim, os nervos ópticos que saem da retina temporal (lateral) enviarão a informação para o seu respectivo hemisfério cerebral (ipsilateralmente). Já os nervos que saem da retina nasal (medial) irão alcançar o hemisfério cerebral oposto (contralateral). Os sistemas ipsilateral e contralateral promovem a troca de informação visual, importante para a localização do objeto. Por exemplo, a luz que incide diretamente no olho, irá ativar toda a retina, sendo a informação transmitida integralmente pelos dois nervos ópticos. Já a luz que incidir lateralmente irá ativar apenas a retina temporal de um olho e a retina nasal do outro olho (ou vice-versa). Assim, diferentes combinações de transtornos podem ocorrer nos nervos e tratos ópticos. Por exemplo, lesões nos nervos ópticos do olho esquerdo prejudicam a visão apenas do seu respectivo olho. Já as lesões nos tratos ópticos esquerdos (após o quiasmo óptico) comprometem a visão de ambos os olhos (hemianopsia homônima). 
A passagem da informação pelo Corpo Geniculado Lateral é uma etapa importante no processo visual, pois promove a interação com os outros sentidos sensoriais (exceto o olfato), sincronizando temporal e espacialmente a informação. Ademais, essa região integra as informações dos hemicampos visuais de cada olho, compondo uma representação tridimensional do objeto, importante para determinar a posição, velocidade e direção dos elementos. O Corpo Geniculado Lateral também fornece informações (output) para sincronizar o movimento dos olhos e para ajustar o foco de acordo com a distância, que são essenciais para a leitura.

Todo esse sistema neurovisual é composto principalmente por células magnocelulares e parvocelulares (EVANS, 2001). Grande atenção tem sido dada as vias magnocelulares, que são responsáveis pela informação sobre a localização do estímulo, dotadas de um rápido sistema de alerta e direcionadas da atenção para novos objetos, pois são sensíveis ao movimento e ao contraste luminoso. Durante a leitura, essas vias mediam a capacidade de identificar rapidamente as letras e sua ordem sequencial, controlam a orientação visual da atenção e das fixações e ainda a sincronização ocular. Para uma melhor performance na atividade magnocelular durante a leitura, tem sido recomendada a ingestão de ômega-3 e ômega-6 na alimentação diária (CYHLAROVA et al., 2007; STEIN, 2014).

A área visual primária se organiza retinotopicamente, ou seja, cada parte da retina é processada por uma região específica do córtex visual. Assim, as áreas periféricas da retina se conectam às regiões mais anteriores do córtex, as áreas centrais ao polo occipital, as áreas superiores à borda superior e as áreas inferiores à borda inferior do sulco calcarino. Finalmente, a partir do momento em que todas essas informações visuais são processadas pelo córtex visual primário é que se pode realmente se falar em visão, já que até então a informação era apenas modulada, mas não elaborada cognitivamente. Como se pode ver, diversos nervos, tratos, regiões cerebrais e tipos de células estão envolvidos na visão e podem acarretar distintos problemas durante a leitura.

\subsection{Processamento da leitura no sistema neuronal da linguagem}

Após os impulsos nervosos visuais chegarem à região occipital (região visual primária), a informação do texto é processada em suas características visuais e posteriormente analisada letra por letra (aproximadamente 27 unidades de letras). A 
partir dessa análise, a informação poderá ser redistribuída para o Giro Angular ou para o Giro Fusiforme Esquerdo, dando início ao processamento do sistema neuronal da linguagem (ver Figura 1) por meio da rota fonológica ou da rota ortográfica lexical, respectivamente (COLTHEART et al., 2001).

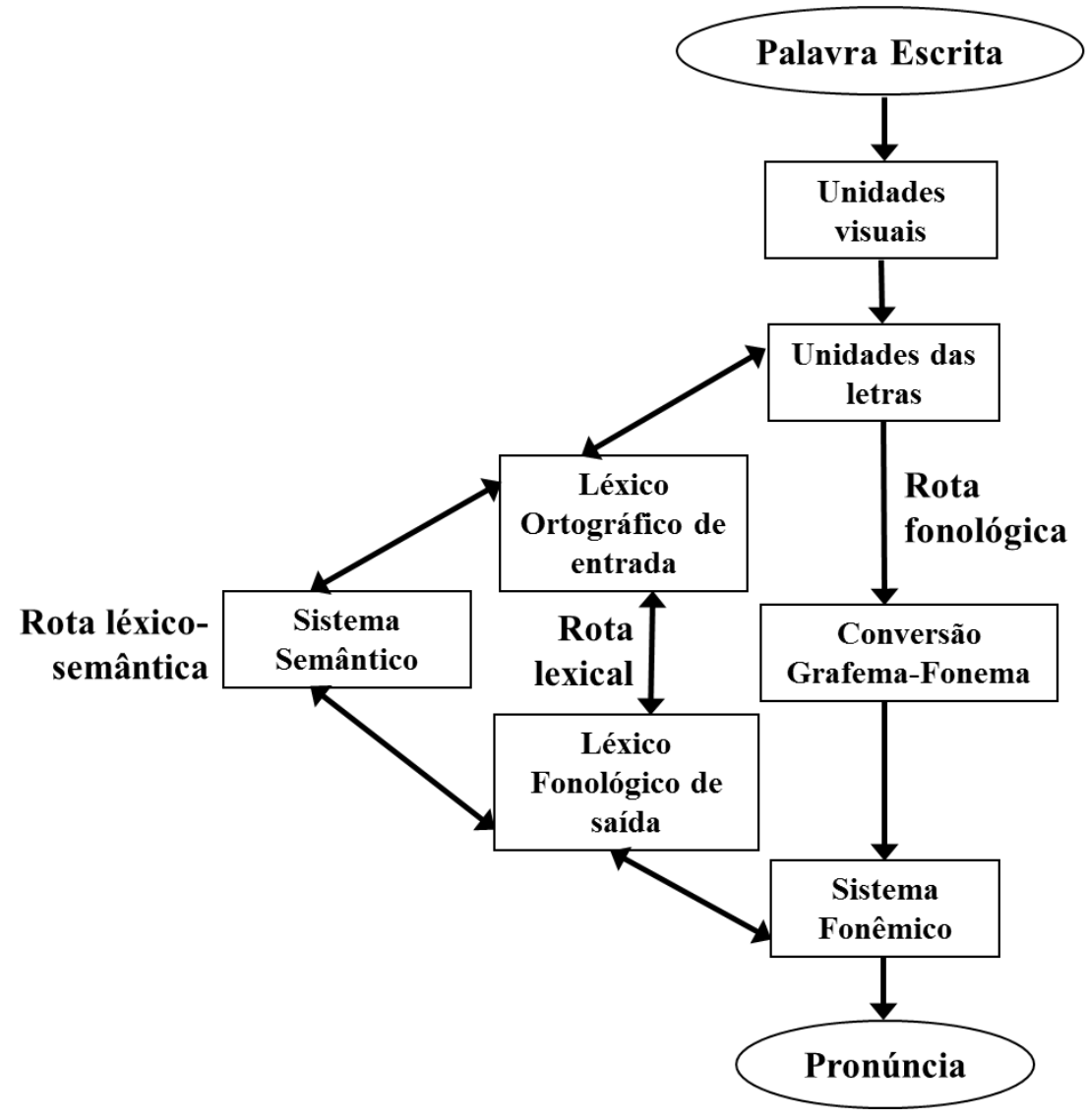

Figura 1. Modelo de dupla rota em cascata de reconhecimento visual de palavras e de leitura em voz alta [adaptado de COLTHEART et al. (2001)]

O giro angular processa a rota fonológica, que é um sistema rudimentar de leitura, necessário para decodificar palavras desconhecidas ou pouco familiares ao leitor. Esse moroso sistema realiza a conversão grafema-fonema, ou seja, transforma as unidades da palavra escrita (ex., b, j, nh, ch) em seu respectivo fonemas (ex., /b/, /3/, $/ \mathrm{n} /, / / /)$. Assim, o leitor deverá integrar cada um dos sons para conseguir pronunciar de forma completa a palavra. Essa rota de leitura é a mais utilizada durante o processo de alfabetização e na leitura de pseudopalavras, e deverá estar consolidada para que a criança se torne uma boa leitora. Intervenções para melhorar a performance da rota fonológica envolvem o ensino explicito do princípio alfabético, o conhecimento dos sons das letras e os jogos de consciência fonológica, como geração (ataque, rima, 
fonema), detecção, síntese, segmentação, supressão e substituição de fonemas (DUFF; CLARKE, 2011).

Já o Giro Fusiforme Esquerdo processa a rota ortográfica lexical, que é um sistema de leitura mais eficiente. Essa região, que também é chamada de caixa de palavras, armazena a organização visual de aproximadamente 8000 palavras inteiras (léxico ortográfico), o que possibilita uma leitura automatizada e fluida, essencial para a leitura de palavras irregulares. No entanto, antes da alfabetização essa região era especializada no reconhecimento de faces de pessoas e objetos. Como o Giro Fusiforme Esquerdo possui neurônios que não diferem a orientação do objeto (se ele está virado para cima, para baixo ou para um dos lados), não seria inicialmente uma região ideal para suportar o armazenamento de palavras, pois estas possuem traços invariantes (a orientação espacial específica da letra é crucial para o seu reconhecimento) (SCLIARCABRAL, 2014). Contudo, os neurônios do Giro Fusiforme Esquerdo modificam sua estrutura, durante o processo de alfabetização, e perdem a capacidade de simetrizar a imagem, sendo agora capazes de armazenar um amplo conjunto visual de palavras. DEHAENE (2012) defende que crianças disléxicas precisam de mais tempo para que essa diferenciação neuronal aconteça. Intervenções para estimular a caixa de palavras envolvem a leitura de textos, a frequente repetição das palavras escritas novas e das palavras irregulares.

Essas duas rotas (fonológica e lexical) de reconhecimento de palavras escritas precisam se conectar de forma robusta com as regiões da linguagem falada, existentes antes do processo de alfabetização. Esse é um dos maiores triunfos da adaptação neuronal, pois a palavra escrita pode se ligar ao seu significado, sintaxe, prosódia, e a produção da fala, do discurso, da inferência e da escrita.

\subsection{Distinção entre transtorno neurovisual e de leitura}

Tanto os transtornos neurovisuais quanto o transtorno específico de leitura (dislexia) são disfunções de base neuronal que afetam a aprendizagem da leitura. Como elucidado neste trabalho, os transtornos neurovisuais podem ocorrer desde o impulso nervoso emitido pela retina até a região visual primária. Ou seja, a dificuldade de leitura pode preceder a etapa de processamento neuronal da linguagem, tendo então como base o processamento visual.

Dentre as disfunções neurovisuais que afetam a qualidade da leitura, a Síndrome de Irlen é a com maior prevalência na população, variando de intensidade em 
cerca de 19-36\% da população geral, com 2-5\% apresentando sintomas severos (WILKINS et al., 2001; EVANS; JOSEPH, 2002; HOLLIS; ALLEN, 2006; NICHOLS et al., 2009). Na literatura, a mesma sintomatologia é encontrada sob diferentes nomes: Síndrome de Meares-Irlen, Síndrome de Sensibilidade Escotópica e Estresse Visual. Essa síndrome é caracterizada principalmente pelo déficit no processamento das informações visuais que leva a: 1) fotofobia: alta sensibilidade a luz, como o brilho do papel branco ou do computador; 2) distorções visuo-perceptuais: percepção que as letras se movem, vibram, aglomeram, e se espelham; 3) limitações na abrangência focal: diminuição do número de letras lidas a cada fixação ocular; 4) dificuldade em manter a atenção visual; e 5) dificuldade na percepção de profundidade (IRLEN, 1983; ROBINSON; MILES, 1987; LOEW; WATSON, 2013). A sua intensidade é afetada pela exposição a certos tipos de iluminação, intensidade, contraste, comprimentos de onda e frequências espaciais. Sinais da Síndrome de Irlen são o hábito de ler à meia-luz, perda do local durante a leitura, frequente ofuscamento, prurido ocular (coceira), ardência, lacrimejamento e cansaço visual crescente.

Por outro lado, a dislexia é causada pela fraca conectividade do sistema neuronal da linguagem (RAMUS, 2014), que acarreta problemas cognitivos principalmente na já referida rota fonológica (consciência fonológica, conversão grafema-fonema, memória de trabalho fonológica) e/ou na rota lexical (reconhecimento automatizado das palavras irregulares) (BLAZELY et al., 2005; ZIEGLER et al., 2008; WYBROW; HANLEY, 2015). Seus sintomas são percebidos precocemente na criança, inclusive antes do processo de alfabetização, por meio de erros sintáticos na fala, fraca nomeação e consciência fonológica (CATTS et al., 2013).

Tanto o Manual de Diagnóstico e Estatística dos Transtornos Mentais (DSM-5) (AMERICAN PSYCHIATRIC ASSOCIATION, 2013) quanto a Classificação Estatística Internacional de Doenças e Problemas Relacionados com a Saúde (CID-10) (ORGANIZAÇÃO MUNDIAL DE SAÚDE, 2004) especificam que a dislexia não pode ser atribuída a problemas na acuidade visual. Apesar de correta, essa especificação diagnóstica é limitada, uma vez que a visão vai muito além da resolução espacial (nitidez), já que os exames oftalmológicos clássicos (ex., refracionais e binocularidade) se restringem ao globo ocular. Assim, para o diagnóstico diferencial da dislexia, é essencial ir além da acuidade visual e incluir exames neurovisuais, como o de sensibilidade ao contraste, estímulos de dupla frequência temporal, oculomotricidade, campo visual periférico dinâmico e estereopsia. 
Outras importantes distinções entre esses dois transtornos estão no tratamento e no prognóstico. Na Síndrome de Irlen, os tratamentos são mais objetivos, pois com o uso de lâminas e lentes espectrais seletivas, que fazem o bloqueio das faixas identificadas como hipersensibilizantes, há uma melhora imediata na fluência e movimentação sacádica da leitura, caso o paciente já seja alfabetizado (SOLAN et al., 1998; ALLEN et al., 2012). Essas intervenções possuem bom prognóstico por aprimorarem as funções das vias neurovisuais magnocelulares, quando usadas de forma permanente (TYRRELL et al., 1995; RAY et al., 2005; NEWMAN WRIGHT et al., 2007). NOBLE et al. (2004) demonstraram que o uso de lâminas espectrais permitiram que crianças com Síndrome de Irlen alcançassem a leitura de seus pares em 3 meses, com o grupo controle apresentando ganhos negligenciáveis. Ademais, ao atenuar a sobrecarga na aquisição visual estes recursos habitualmente potencializam as demais intervenções quando coexistem outros transtornos.

Já os disléxicos, em especial os de grau mais severo, normalmente passam por longos períodos de acompanhamento multidisciplinar focados no aprimoramento da consciência fonológica por meio de métodos multissensoriais. O prognóstico é menos previsível, uma vez que a dislexia é vitalícia, e assim, mesmo nos pacientes em que as intervenções educacionais eliminaram os sintomas comportamentais do transtorno (disléxicos compensados), a atividade neuronal continuará distinta do leitor com desenvolvimento típico (PAULESU et al., 1996). Estudos demonstraram que 43-53\% das crianças e adultos com dislexia são beneficiadas pelo uso das lâminas espectrais, que é um dos indicadores diagnósticos da Síndrome de Irlen (KRISS; EVANS, 2005; SINGLETON; HENDERSON, 2007; NICHOLS et al., 2009).

A Tabela 1 sumariza as características físicas, educacionais e psicossociais da Síndrome de Irlen e da Dislexia, com o intuito de auxiliar no diagnóstico diferencial. Essas características devem ser analisadas como fatores de risco e nunca de forma determinista, uma vez que tanto a Síndrome de Irlen quanto a Dislexia se manifestam de forma heterogênea e com diferentes intensidades (espectro de dificuldades). Ademais, deve-se considerar a possível coexistência entre os transtornos, como Transtorno do Espectro Autista, Transtorno de Déficit de Atenção/Hiperatividade, Transtornos Disruptivos. 
Tabela 1. Caraterísticas em comum e específicas da Síndrome de Irlen e da Dislexia

\begin{tabular}{|c|c|c|}
\hline CARACTERISSTICAS & $\begin{array}{c}\text { SÍNDROM } \\
\text { E DE } \\
\text { IRLEN }\end{array}$ & DISLEXIA \\
\hline \multicolumn{3}{|l|}{ Sintomas físicos } \\
\hline Ardência, coceira ou lacrimejamento nos olhos & $\mathrm{X}$ & \\
\hline Fricciona ou esfrega os olhos frequentemente & $\mathrm{X}$ & \\
\hline Pisca os olhos excessivamente & $\mathrm{X}$ & \\
\hline Dores de cabeça, dores de estômago ou fadiga & $\mathrm{X}$ & $\mathrm{X}$ \\
\hline Protege os olhos da luz durante a leitura & $\mathrm{X}$ & \\
\hline Inclina a cabeça durante a leitura & $\mathrm{X}$ & \\
\hline $\begin{array}{l}\text { Rápidos sintomas de fadiga durante a leitura }( \pm 15 \\
\text { minutos) }\end{array}$ & $\mathrm{X}$ & \\
\hline \multicolumn{3}{|l|}{ Padrão durante a leitura } \\
\hline $\begin{array}{l}\text { Lentidão no aprendizado da conexão entre letras } \\
\text { individuais e sons (grafema-fonema) }\end{array}$ & & $\mathrm{X}$ \\
\hline Vê textos com distorções e instabilidade & $\mathrm{X}$ & \\
\hline Perde facilmente a localização do que está lendo & $\mathrm{X}$ & $\mathrm{X}$ \\
\hline Pula palavras ou linhas & $\mathrm{X}$ & $\mathrm{X}$ \\
\hline Leitura silabada & & $\mathrm{X}$ \\
\hline Faz leituras com lentidão & $X$ & $\mathrm{X}$ \\
\hline Mistura números em exercícios de matemática & $\mathrm{X}$ & $\mathrm{X}$ \\
\hline Evita a leitura ou a interrompe frequentemente & $\mathrm{X}$ & $\mathrm{X}$ \\
\hline Prefere leitura em ambiente com pouca luz & $\mathrm{X}$ & \\
\hline Pouca compreensão do que está lendo & $\mathrm{X}$ & $\mathrm{X}$ \\
\hline \multicolumn{3}{|l|}{ Percepção auditiva e produção da fala } \\
\hline Atraso para aprender a falar e linguagem verbal imatura & & $\mathrm{X}$ \\
\hline Dificuldade em escutar e compreender as palavras & & $\mathrm{X}$ \\
\hline $\begin{array}{l}\text { Dificuldade para seguir instruções verbais dadas } \\
\text { rapidamente }\end{array}$ & & $\mathrm{X}$ \\
\hline Mistura sílabas e erros sintáticos na fala & & $\mathrm{X}$ \\
\hline Fraca consciência fonológica & & $\mathrm{X}$ \\
\hline Dificuldade em juntar sons para formar palavras & & $\mathrm{X}$ \\
\hline Dificuldade para perceber ou produzir rimas & & $\mathrm{X}$ \\
\hline Dificuldades em nomear objetos familiares & & $\mathrm{X}$ \\
\hline \multicolumn{3}{|c|}{ Escrita, caligrafia e comportamento físico } \\
\hline Tamanho desigual de letra ou espaçamento & $\mathrm{X}$ & $\mathrm{X}$ \\
\hline Escreve as palavras de cabeça para baixo ou espelhadas & & $\mathrm{X}$ \\
\hline Inversão da posição das letras, sílabas ou palavras & & $\mathrm{X}$ \\
\hline Dificuldade para escrever nas linhas & $\mathrm{X}$ & $\mathrm{X}$ \\
\hline $\begin{array}{l}\text { Predisposição a caligrafia ruim; evita escrever com letra } \\
\text { cursiva }\end{array}$ & $\mathrm{X}$ & $\mathrm{X}$ \\
\hline $\begin{array}{l}\text { Dificuldade para controlar o lápis para escrever ou } \\
\text { desenhar }\end{array}$ & & $\mathrm{X}$ \\
\hline Ser ambidestro (lentidão em definir uma das mãos) & & $\mathrm{X}$ \\
\hline Escrever com a mão esquerda & & $\mathrm{X}$ \\
\hline Fazer confusão entre esquerda e direita & & $\mathrm{X}$ \\
\hline $\begin{array}{l}\text { Ser desastrado, propensão a quedas e acidentes } \\
\text { domésticos }\end{array}$ & $\mathrm{X}$ & $\mathrm{X}$ \\
\hline
\end{tabular}




\begin{tabular}{|l|c|c|}
\hline \multicolumn{2}{|c|}{ Atividades e Psicossocial } & $\mathrm{X}$ \\
\hline Dificuldade para aprender a dizer as horas & $\mathrm{X}$ \\
\hline $\begin{array}{l}\text { Dificuldade com sequenciamento (ex., alfabeto, meses } \\
\text { do ano) }\end{array}$ & $\mathrm{X}$ & $\mathrm{X}$ \\
\hline Dificuldade para finalizar os deveres de casa & $\mathrm{X}$ & $\mathrm{X}$ \\
\hline Histórico familiar de dislexia & $\mathrm{X}$ & $\mathrm{X}$ \\
\hline $\begin{array}{l}\text { Histórico familiar de fadiga com a leitura, sensibilidade } \\
\text { à luz, dores de cabeça e enxaqueca }\end{array}$ & $\mathrm{X}$ & $\mathrm{X}$ \\
\hline Inquietude ou falta de atenção & $\begin{array}{c}\text { Lâminas e } \\
\text { filtros } \\
\text { espectrais }\end{array}$ & $\begin{array}{c}\text { Consciênci } \\
\text { a } \\
\text { fonológica } \\
\text { e treino } \\
\text { lexical }\end{array}$ \\
\hline Predisposição à depressão, frustração ou raiva & \\
\hline
\end{tabular}

Fonte: Elaboração própria

\section{CONCLUSÃO}

A leitura é uma habilidade essencial no mundo moderno, tendo todos o direito a aprender a ler, independentemente de sua capacidade e oportunidade biopsicossocial. Para isso, é necessário o estudo da relação de todos os sistemas que compõem o processo de leitura, integrando o processamento óptico, o neurovisual e o cognitivo. Distintos transtornos nesses sistemas podem causar problemas de leitura, no entanto o diagnóstico diferencial deve ser feito para que haja o adequado tratamento e prognóstico.

\section{REFERÊNCIAS}

ALLEN, P. M. et al. Accommodation, pattern glare, and coloured overlays. Perception, Londres, v. 41, n. 12, p. 1458-1467, 2012.

AMERICAN PSYCHIATRIC ASSOCIATION. Diagnostic and statistical manual of mental disorders $5^{\text {th }}$ edition: DSM-5. Washington, D.C.: American Psychiatric Association, 947 p., 2013.

BLAZELY, A. M.; COLTHEART, M.; CASEY, B. J. Semantic impairment with and without surface dyslexia: Implications for models of reading. Cognitive Neuropsychology, London, v. 22, n. 6, p. 695-717, 2005.

BORRELLI, M. et al. Avaliação da qualidade da visão, na prática da leitura diária, em relação à formatação dos textos. Revista Brasileira de Oftalmologia, Rio de Janeiro, v. 69, p. 114-120, 2010.

CATTS, H. W. et al. Early Identification of Reading Disabilities Within an RTI Framework. Journal of Learning Disabilities, Chicago, v. 48, n. 3, p.281-297, 2013.

CIUFFREDA, K. J.; KENYON, R. V.; STARK, L. Saccadic intrusions contributing to reading disability: a case report. American Journal of Optometry and Physiological optics, Chicago, v. 60, n. 3, p. 242-249, 1983.

COLTHEART, M. et al. DRC: a dual route cascaded model of visual word recognition and reading aloud. Psychological Review, Washington, v. 108, n. 1, p. 204-256, 2001.

COSENZA, R. M. Fundamentos de Neuroanatomia. Rio de Janeiro: Guanabara Koogan, v. 3, 2005. 
CYHLAROVA, E. et al. Membrane fatty acids, reading and spelling in dyslexic and non-dyslexic adults. European Neuropsychopharmacology, Amsterdam, v. 17, n. 2, p. 116-121, 2007.

DEHAENE, S. Os neurônios da leitura: Como a ciência explica a nossa capacidade de ler. Porto Alegre: Penso, 2012.

DUFF, F. J.; CLARKE, P. J. Practitioner Review: Reading disorders: what are the effective interventions and how should they be implemented and evaluated? Journal of Child Psychology and Psychiatry, Oxford, v. 52, n. 1, p. 3-12, 2011.

EVANS, B. Dyslexia and Vision. Michigan: Wiley, 2001.

EVANS, B. J.; JOSEPH, F. The effect of coloured filters on the rate of reading in an adult student population. Ophthalmic and Physiological Optics, Oxford, v. 22, n. 6, p. 535-545, 2002.

FELLEMAN, D. J.; VAN ESSEN, D. C. Distributed hierarchical processing in the primate cerebral cortex. Cerebral Cortex, New York, v. 1, n. 1, p. 1-47, 1991.

HOLLIS, J.; ALLEN, P. M. Screening for Meares-Irlen sensitivity in adults: can assessment methods predict changes in reading speed? Ophthalmic and Physiological Optics, Oxford, v. 26, n. 6, p. 566-571, 2006.

IRLEN, H. Successful treatment of leaning disabilities. Annual Meeting of the American Psychological Association, Anaheim, 1983.

KRISS, I.; EVANS, B. J. W. The relationship between dyslexia and Meares-Irlen Syndrome. Journal of Research in Reading, Leeds, v. 28, n. 3, p. 350-364, 2005.

LEONG, D. F. et al. The Effect of Saccadic Training on Early Reading Fluency. Clinical Pediatrics, Philadelphia, v. 53, n. 9, p. 858-864, 2014.

LIGHTSTONE, A.; LIGHTSTONE, T.; WILKINS, A. J. Both coloured overlays and coloured lenses can improve reading fluency, but their optimal chromaticities differ. Ophthalmic and Physiological Optics, Oxford, v. 19, n. 4, p. 279-285, 1999.

LIN, L. L. et al. Epidemiologic study of ocular refraction among schoolchildren in Taiwan in 1995. Optometry and Vision Science, Baltimore, v. 76, n. 5, p. 275-281, 1999.

LOEW, S. J.; WATSON, K. The prevalence of symptoms of scotopic sensitivity/MearesIrlen syndrome in subjects diagnosed with ADHD: Does misdiagnosis play a significant role? Hrvatska Revija za Rehabilitacijska Istraživanja, Zagreb, v. 49, p. 64-72, 2013.

MANSFIELD, J. S.; LEGGE, G. E.; BANE, M. C. Psychophysics of reading. XV: Font effects in normal and low vision. Investigative Ophthalmology \& Visual Science, St. Louis, v. 37, n. 8, p. 1492-1501, 1996.

MCGUINNESS, D. O ensino da leitura: o que a ciência nos diz sobre como ensinar a ler. Porto Alegre: Artmed, 2006.

MIRSHAHI, A. et al. Myopia and Level of Education. Ophthalmology, Rochester, v. 121, n. 10, p. 2047-2052, 2014.

NEWMAN WRIGHT, B.; WILKINS, A.; ZOUKOS, Y. Spectral filters can improve reading and visual search in patients with multiple sclerosis. Journal of Neurology, Berlin, v. 254, n. 12, p. 1729-1735, 2007.

NICHOLS, S. A. et al. Screening for dyslexia, dyspraxia and Meares-Irlen syndrome in higher education. Dyslexia, Chichester, v. 15, n. 1, p. 42-60, 2009.

NOBLE, J. et al. A controlled field study of the use of coloured overlays on reading achievement. Australian Journal of Learning Difficulties, Abingdon, v. 9, n. 2, p. 14-22, 2004. 
ORGANIZAÇÃO MUNDIAL DE SAÚDE. International statistical classification of diseases and related health problems. 10th revision, 2nd edition. Geneva: World Health Organization, 2004.

PAULESU, E. et al. Is developmental dyslexia a disconnection syndrome? Brain, Oxford, v. 119, n. 1, p. 143-157, 1996.

RAMUS, F. Neuroimaging sheds new light on the phonological deficit in dyslexia. Trends in Cognitive Sciences, Kidlington, v. 18, n. 6, p. 274-275, 2014.

RAY, N. J.; FOWLER, S.; STEIN, J. F. Yellow Filters Can Improve Magnocellular Function: Motion Sensitivity, Convergence, Accommodation, and Reading. Annals of the New York Academy of Sciences, New York, v. 1039, n. 1, p. 283-293, 2005.

ROBINSON, G. L.; MILES, J. The use of coloured overlays to improve visual processing - a preliminary survey. International Journal of Disability, Development and Education, London, v. 34, n. 1, p. 65-70, 1987.

SCLIAR-CABRAL, L. Neuron recycling for learning the alphabetic principles. Folia Phoniatrica et Logopaedica, Basel, v. 66, n. 1-2, p. 58-66, 2014.

SHERWIN, J. C. et al. The association between time spent outdoors and myopia in children and adolescents: a systematic review and meta-analysis. Ophthalmology, Rochester, v. 119, n. 10, p. 2141-2151, 2012.

SINGLETON, C.; HENDERSON, L.-M. Computerized screening for visual stress in children with dyslexia. Dyslexia, Chichester, v. 13, n. 2, p. 130-151, 2007.

SOLAN, H. A. et al. Eye movement efficiency in normal and reading disabled elementary school children: effects of varying luminance and wavelength. Journal of the American Optometric Association, St. Louis, v. 69, n. 7, p. 455-464, 1998.

STEIN, J. Dyslexia: the Role of Vision and Visual Attention. Current Developmental Disorders Reports, Cham, v. 1, n. 4, p. 267-280, 2014.

TAYLOR ASSOCIATES. Visagraph III: resource guide. New York, 2006.

TYRRELL, R. et al. Coloured overlays, visual discomfort, visual search and classroom reading. Journal of Research in Reading, London, v. 18, n. 1, p. 10-23, 1995.

VILHENA, D. A. A evolução da escrita e a adaptação do cérebro. In: PEREIRA, R. S. (Ed.). Abordagem Multidisciplinar da Aprendizagem. 1. Sacavém, Portugal: QualConsoante Lda, p. 241-251, 2015.

VITALE, S.; SPERDUTO, R. D.; FERRIS, F. L., 3RD. Increased prevalence of myopia in the United States between 1971-1972 and 1999-2004. Archives of Ophthalmology, Chicago, v. 127, n. 12, p. 1632-1639, 2009.

WILKINS, A. J. et al. Coloured overlays and their benefit for reading. Journal of Research in Reading, Leeds, v. 24, n. 1, p. 41-64, 2001.

WYBROW, D. P.; HANLEY, J. R. Surface developmental dyslexia is as prevalent as phonological dyslexia when appropriate control groups are employed. Cognitive Neuropsychology, London, v. 32, n. 1, p. 1-13, 2015.

ZIEGLER, J. C. et al. Developmental dyslexia and the dual route model of reading: simulating individual differences and subtypes. Cognition, Amsterdam, v. 107, n. 1, p. 151-178, 2008. 